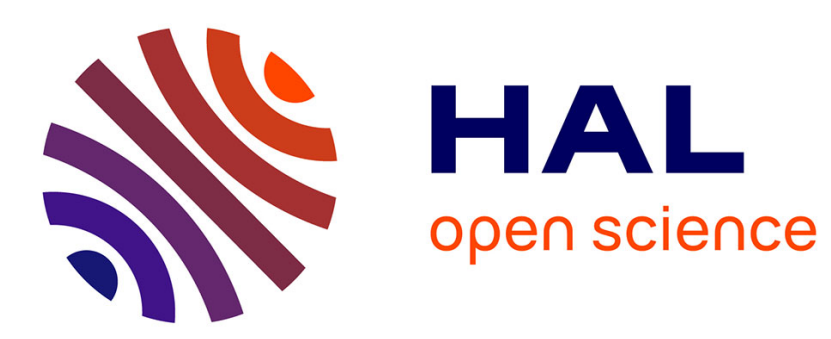

\title{
Numerical and analytical calculation of modal excitability for elastic wave generation in lossy waveguides
}

Fabien Treyssède, Laurent Laguerre

\section{- To cite this version:}

Fabien Treyssède, Laurent Laguerre. Numerical and analytical calculation of modal excitability for elastic wave generation in lossy waveguides. Journal of the Acoustical Society of America, 2013, 133, pp.3827-3837. hal-01066393

\section{HAL Id: hal-01066393 \\ https://hal.science/hal-01066393}

Submitted on 22 Sep 2014

HAL is a multi-disciplinary open access archive for the deposit and dissemination of scientific research documents, whether they are published or not. The documents may come from teaching and research institutions in France or abroad, or from public or private research centers.
L'archive ouverte pluridisciplinaire HAL, est destinée au dépôt et à la diffusion de documents scientifiques de niveau recherche, publiés ou non, émanant des établissements d'enseignement et de recherche français ou étrangers, des laboratoires publics ou privés. 


\title{
Numerical and analytical calculation of modal excitability for elastic wave generation in lossy waveguides
}

Fabien Treyssède ${ }^{\mathrm{a})}$ and Laurent Laguerre

LUNAM Université, IFSTTAR, route de Bouaye, 44344 Bouguenais, France

(Dated: March 22, 2013)

\begin{abstract}
In the analysis of elastic waveguides, the excitability of a given mode is an important feature defined by the displacement-force ratio. Useful analytical expressions have been provided in the literature for modes with real wavenumbers (propagating modes in lossless waveguides). The central result of this paper consists in deriving a generalized expression for the modal excitability valid for modes with complex wavenumbers (lossy waveguides or non-propagating modes). The analysis starts from a semi-analytical finite element method and avoids solving the left eigenproblem. Analytical expressions of modal excitability are then deduced. It is shown that the fundamental orthogonality property to be used indeed corresponds to a form of Auld's real orthogonality relation, involving both positive and negative-going modes. Finally, some results obtained from the generalized excitability are compared to the approximate lossless expression.
\end{abstract}

PACS numbers: 43.35.Cg, 43.20.Mv, 43.20.Bi

\section{INTRODUCTION}

Elastic guided waves are are of great interest in the context of non-destructive testing of elongated structures. Such waves are dispersive and multimodal, which complicates their practical use. Dispersion curves of phase and group velocities as functions of frequency are useful to identify modes that propagate in a frequency range with low dispersion and low attenuation ${ }^{1}$. Such curves represent modal properties obtained regardless excitation. Yet for a practical inspection system, it is also essential to determine and control the amplitudes of each guided modes excited by a given source. Such an information typically allows to optimize the type and location of sensors to be used.

Two approaches can mainly been distinguished in order to calculate the response of waveguides under excitation. A first approach consists in using integral transform techniques ${ }^{2-5}$ With this method, the response is obtained by a contour integration in the complex plane or numerical integration of the analytical solution explicitly derived in the wavenumber domain.

An alternative approach is based on modal analysis, which consists in expanding the excited field as a sum of guided modes ${ }^{6-8}$. The contribution of each mode is obtained in terms of the associated power flow from an orthogonality relation between eigenmodes. This second method appears to be more suitable to achieve a better interpretation and optimization of signals, as it directly provides the contribution of each modes as a function of the excitation. In particular, this method has allowed to introduce the modal excitability ${ }^{9-11}$. For a given frequency, the excitability of a particular mode can be defined as the ratio of the displacement of that mode to a point force applied in a given direction.

\footnotetext{
a) Electronic address: fabien.treyssede@ifsttar.fr
}

In practice, many applications involve lossy structures (e.g. made of viscoelastic materials). While the first approach has readily been applied to lossy waveguides $^{12,13}$, analytical derivations of modal expansions use the so-called Auld's complex orthogonality relation, limited to real wavenumbers only ${ }^{6}$ (propagating modes in lossless waveguides). Hence, neither nonpropagating modes nor viscoelastic materials can be considered, although Auld's relation is sometimes applied as a first approximation ${ }^{14-16}$.

In addition to the above mentioned results, which are essentially analytical, the modal approach has also been applied on the basis of a numerical method referred to as the semi-analytical finite element (SAFE) method. The SAFE method is particularly efficient from a computational point of view because the axial dependence $e^{i k z}$ is assumed before finite element (FE) discretization $(k$ and $z$ denote the wavenumber and distance along the waveguide axis, respectively). As a consequence, only the transverse dimensions need to be discretized and meshed - see for instance Refs. [17-19]. The waveguide response can be computed thanks to finite element eigenvectors orthogonality resulting from properties of the matrix eigensystem ${ }^{20,21}$. The advantage of SAFE methods is their capacity to handle complex waveguides of arbitrary cross-section, together with material anisotropy and transversely inhomogeneous properties. Interestingly, viscoelastic materials can also be handled with the introduction of left eigenvectors of the matrix eigensystem ${ }^{22,23}$.

In this paper, one shows how to simply compute the modal excitability from the numerical SAFE method and highlights the direct link with analytical expressions. In particular, the central result of this paper provides a generalized expression for the modal excitability, which remains valid for lossy waveguides as well as non-propagating modes in lossless waveguides. Due to its relative simplicity, the approach adopted in this work consists in starting from the SAFE method and then de- 
ducing the analytical expressions from the finite element ones.

Section II briefly recalls the SAFE formulation, as standardly presented in the literature. The concept of eigenforces is introduced, which will greatly simplify the interpretation of SAFE equations and their link with the analytical results in subsequent sections. In Sec. III, a SAFE version of the lossless analytical excitability is derived for lossless waveguides, which highlights the direct link between numerical and analytical approaches. In Sec. IV, the excitability expression is modified and generalized to non-propagating modes and lossy waveguides. Section V finally gives illustrative examples. Results obtained with the lossless and the generalized excitabilities are compared.

\section{SAFE FORMULATION}

One assumes a linearly elastic material, small strains and displacements with a time harmonic $e^{-i \omega t}$ dependence. For clarity, the variational formulation of elastodynamics in the frequency domain is given by:

$\int_{V} \delta \boldsymbol{\epsilon}^{T} \boldsymbol{\sigma} d V-\omega^{2} \int_{V} \rho \delta \mathbf{u}^{T} \mathbf{u} d V=\int_{V} \delta \mathbf{u}^{T} \mathbf{f} d V+\int_{\partial V} \delta \mathbf{u}^{T} \mathbf{t} d \partial V$

where $\mathbf{u}, \boldsymbol{\epsilon}$ and $\boldsymbol{\sigma}$ are the displacement, strain and stress vectors. $\rho$ is the material density. $\delta$ denotes virtual fields. The stress-strain relation is $\boldsymbol{\sigma}=\mathbf{C} \boldsymbol{\epsilon}$, where $\mathbf{C}$ is the matrix of material properties. $\mathbf{C}$ is complex in the case of viscoelastic materials. f represents acoustic sources inside the structural volume $V$. $\mathbf{t}$ is the traction vector applied on the surface $\partial V$. In index notations, t is related to the stress tensor by $t_{i}=\sigma_{i j} n_{j}$, where $n_{j}$ is the outward normal to the boundary $\partial V$. For clarity, the strain and stress vectors for three-dimensional problems are: $\boldsymbol{\epsilon}=\left[\begin{array}{llllll}\epsilon_{x x} & \epsilon_{y y} & \epsilon_{z z} & 2 \epsilon_{x y} & 2 \epsilon_{x z} & 2 \epsilon_{y z}\end{array}\right]^{T}$ and $\boldsymbol{\sigma}=\left[\begin{array}{llllll}\sigma_{x x} & \sigma_{y y} & \sigma_{z z} & \sigma_{x y} & \sigma_{x z} & \sigma_{y z}\end{array}\right]^{T}$.

In this section, the SAFE technique is briefly recalled. For more details on this method, the reader can refer to $[17-19,22-25]$ for instance.

Also, the concept of eigenforces is introduced. Eigenforces are defined as modal forces associated with displacement modeshapes.

\section{A. Governing equations}

Let us denote $z$ the axis of the waveguide, $x$ and $y$ the transverse coordinates. Separating transverse from axial derivatives, the strain-displacement relation can be rewritten as: $\boldsymbol{\epsilon}=\left(\mathbf{L}_{x y}+\mathbf{L}_{z} \partial / \partial z\right) \mathbf{u}$, where $\mathbf{L}_{x y}$ is the operator containing all terms except derivatives with respect to $z$.

The SAFE technique consists in starting from a variational formulation integrated on the cross-section $S$, instead of the formulation (1) integrated on the volume $V$ $(d V=d S d z)$. After finite element discretization, the SAFE approach yields the matrix system:

$$
\mathbf{K}_{1} \mathbf{U}-\omega^{2} \mathbf{M U}+\left(\mathbf{K}_{2}-\mathbf{K}_{2}^{T}\right) \mathbf{U}_{, z}-\mathbf{K}_{3} \mathbf{U}_{, z z}=\mathbf{F}
$$

with the following elementary matrices:

$$
\begin{aligned}
& \mathbf{K}_{1}^{e}=\int_{S^{e}} \mathbf{N}^{e T} \mathbf{L}_{x y}^{T} \mathbf{C} \mathbf{L}_{x y} \mathbf{N}^{e} d S, \mathbf{K}_{2}^{e}=\int_{S^{e}} \mathbf{N}^{e T} \mathbf{L}_{x y}^{T} \mathbf{C L} \mathbf{L}_{z} \mathbf{N}^{e} d S, \\
& \mathbf{K}_{3}^{e}=\int_{S^{e}} \mathbf{N}^{e T} \mathbf{L}_{z}^{T} \mathbf{C} \mathbf{L}_{z} \mathbf{N}^{e} d S, \mathbf{M}^{e}=\int_{S^{e}} \rho \mathbf{N}^{e T} \mathbf{N}^{e} d S
\end{aligned}
$$

On one element $S^{e}$ of the cross-section, the displacement is interpolated as $\mathbf{u}=\mathbf{N}^{e} \mathbf{U}^{e}$, where $\mathbf{N}^{e}$ is the matrix of nodal interpolating functions on the element. Note that $\mathbf{K}_{1}, \mathbf{K}_{3}$ and $\mathbf{M}$ are symmetric.

For clarity, $\mathbf{U}=\mathbf{U}(z, \omega)$ is the column vector containing cross-section nodal displacements in the spacefrequency domain. $\mathbf{F}=\mathbf{F}(z, \omega)$ represents the excitation vector in the space-frequency domain, gathering both contributions of volume sources $\mathbf{f}$ and surface sources $\mathbf{t}$.

In the wavenumber-frequency domain, Eq. (2) becomes:

$$
\left\{\mathbf{K}_{1}-\omega^{2} \mathbf{M}+i k\left(\mathbf{K}_{2}-\mathbf{K}_{2}^{T}\right)+k^{2} \mathbf{K}_{3}\right\} \tilde{\mathbf{U}}=\tilde{\mathbf{F}}
$$

where $\tilde{\mathbf{U}}=\tilde{\mathbf{U}}(k, \omega)$ and $\tilde{\mathbf{F}}=\tilde{\mathbf{F}}(k, \omega)$. The following Fourier spatial transform has been introduced: $\tilde{f}(k)=$ $\int_{-\infty}^{+\infty} f(z) e^{-i k z} d z$.

As standardly done in the SAFE literature, Eq. (4) can be recast into a linear form, for instance:

$$
(\mathbf{A}-k \mathbf{B}) \hat{\mathbf{U}}=\hat{\mathbf{F}}
$$

with:

$$
\mathbf{A}=\left[\begin{array}{cc}
\mathbf{0} & \mathbf{K}_{1}-\omega^{2} \mathbf{M} \\
\mathbf{K}_{1}-\omega^{2} \mathbf{M} & i\left(\mathbf{K}_{2}-\mathbf{K}_{2}^{T}\right)
\end{array}\right], \mathbf{B}=\left[\begin{array}{cc}
\mathbf{K}_{1}-\omega^{2} \mathbf{M} & \mathbf{0} \\
\mathbf{0} & -\mathbf{K}_{3}
\end{array}\right]
$$

and:

$$
\hat{\mathbf{U}}=\left\{\begin{array}{c}
\tilde{\mathbf{U}} \\
k \tilde{\mathbf{U}}
\end{array}\right\}, \hat{\mathbf{F}}=\left\{\begin{array}{c}
\mathbf{0} \\
\tilde{\mathbf{F}}
\end{array}\right\}
$$

\section{B. Eigenvectors}

Given $\omega$ and suppressing excitation, Eq. (5) corresponds to a generalized linear eigenvalue problem for finding the wavenumbers:

$$
\left\{\mathbf{A}-k_{m} \mathbf{B}\right\} \hat{\mathbf{U}}_{m}=\mathbf{0}
$$

where $\hat{\mathbf{U}}_{m}^{T}=\left[\begin{array}{lll}\mathbf{U}_{m}^{T} & k_{m} \mathbf{U}_{m}^{T}\end{array}\right]^{T}$. Such an eigenproblem can be solved by standard numerical solvers ${ }^{26}$, as opposed to the quadratic one that would be obtained from the formulation (4). The eigensolutions $\left(k_{m}, \mathbf{U}_{m}\right)$ represent the wavenumber and the displacement vector associated with the guided mode $m$ (note that tilde on the $\mathbf{U}_{m}$ 's can be dropped for conciseness of notations).

From a mathematical point of view, the $\hat{\mathbf{U}}_{m}$ 's are right eigenvectors. Because the eigenproblem (8) is generally unsymmetric, left eigenvectors are also needed ${ }^{27}$. By definition, the left eigenvectors $\hat{\mathbf{V}}_{n}$ satisfy the following eigenproblem:

$$
\hat{\mathbf{V}}_{n}^{T}\left\{\mathbf{A}-k_{n} \mathbf{B}\right\}=\mathbf{0}
$$


Pre-multiplying Eq. (8) by $\hat{\mathbf{V}}_{n}^{T}$, post-multipying Eq. (9) by $\hat{\mathbf{U}}_{m}$ and subtracting both equations yields the following biorthogonality relations:

$$
\hat{\mathbf{V}}_{n}^{T} \mathbf{B} \hat{\mathbf{U}}_{m}=b_{m} \delta_{m n}, \quad \hat{\mathbf{V}}_{n}^{T} \mathbf{A} \hat{\mathbf{U}}_{m}=k_{m} b_{m} \delta_{m n}
$$

where $b_{m}$ is a normalization factor and $\delta_{m n}$ denotes Kronecker's symbol. The above relations are biorthogonal in the sense that both right and left eigenvectors are involved in the expressions.

\section{Forced response}

The solution to Eq. (5), which is the forced response under the excitation $\hat{\mathbf{F}}$, is now expanded as a sum of guided modes: $\hat{\mathbf{U}}=\sum_{m=1}^{2 M} \alpha_{m} \hat{\mathbf{U}}_{m} \cdot 2 M$ is the number of modes, theoretically equal to twice the number of degrees of freedom (dofs) involved in the system (4), but truncated in practice.

Using this eigenmode expansion into Eq. (5) and taking advantage of biorthogonality relations (10), the $\alpha_{m}$ 's are determined ${ }^{18,23-25}$. The displacement response in the wavenumber-frequency domain is:

$$
\tilde{\mathbf{U}}=\sum_{m=1}^{2 M} \frac{\hat{\mathbf{V}}_{m}^{T} \hat{\mathbf{F}}}{b_{m}\left(k_{m}-k\right)} \mathbf{U}_{m}
$$

Then, one applies the inverse Fourier spatial transform, defined by $f(z)=\frac{1}{2 \pi} \int_{-\infty}^{+\infty} \tilde{f}(k) e^{i k z} d k$. Under the assumption that $\tilde{\mathbf{F}}$ is holomorphic (no pole), Cauchy residue theorem yields the response $\mathbf{U}$ in the spacefrequency domain:

$$
\mathbf{U}=-i \sum_{m=1}^{M} \frac{\hat{\mathbf{V}}_{m}^{+T} \hat{\mathbf{F}}\left(k_{m}^{+}\right) e^{i k_{m}^{+} z}}{b_{m}^{+}} \mathbf{U}_{m}^{+}
$$

for the positive-going wave and for $z$ outside the source region (one assumes that the excitation $\mathbf{F}$ is zero outside a finite interval along $z$ ). If one is interested in the response at a location $z$ inside the source region, the application of residue theorem could still be performed by splitting the integrand into two parts ${ }^{28}$.

As opposed to Eq. (11), note that the summation in Eq. (12) is performed over positive-going modes $\left(k_{m}^{+}, \mathbf{U}_{m}^{+}\right)$only $(m=1, \ldots, M)$. The expression for the negative-going wave is obtained by replacing the superscripts + with - and the $-i$ factor with $+i$ in Eq. (12).

In practice, modal bases can be truncated by retaining modes satisfying $\left|\operatorname{Im}\left(k_{m}^{ \pm} z\right)\right|<\gamma$, where $\gamma$ is a user-defined parameter and $z$ is the position at which the response is calculated. The amplitudes of non-propagating modes not included in the expansion are then attenuated by a factor $e^{\gamma}$ at least.

Finally, the response in the space-time $(z, t)$ domain can be obtained from the inverse time Fourier transform of $\mathbf{U}$, given by $\frac{1}{2 \pi} \int_{-\infty}^{+\infty} \mathbf{U} e^{-i \omega t} d \omega$.

For conciseness, one will consider positive-going waves only and drop the + superscripts in the remainder of the paper.
As a side remark, it is noteworthy that backward modes sometimes occur in elastic waveguides (energy and phase velocities have then opposite signs). Hence, the traveling direction of a mode must be determined from the sign of its energy velocity when propagating, and from the sign of $\operatorname{Im}\left(k_{m}\right)$ when non-propagating.

\section{Definition of eigenforces}

The definition of eigenforces is now briefly introduced. The eigenforce concept has recently been used by the authors in Refs. [29, 30] in the context of scattering of guided waves by inhomogeneities.

The eigenforce vector $\mathbf{F}_{m}$, associated with the displacement modeshape $\mathbf{U}_{m}$, can be defined from:

$$
\int_{S} \delta \mathbf{u}^{T} \mathbf{t}_{m} d S=\delta \mathbf{U}^{T} \mathbf{F}_{m}
$$

where $\mathbf{t}_{m}$ is the traction vector applied on the crosssection $S$, whose unit normal is $\mathbf{e}_{z}$. Then, it can be checked that $\mathbf{t}_{m}=\mathbf{L}_{z}^{T} \boldsymbol{\sigma}_{m}=\mathbf{L}_{z}^{T} \mathbf{C}\left(\mathbf{L}_{x y}+i k_{m} \mathbf{L}_{z}\right) \mathbf{u}_{m}$, so that modal forces can be explicitly calculated from displacements by:

$$
\mathbf{F}_{m}=\left(\mathbf{K}_{2}^{T}+i k_{m} \mathbf{K}_{3}\right) \mathbf{U}_{m}
$$

As shown in the present paper, the introduction of modal forces will greatly simplify the interpretation of SAFE equations and their link with analytical results of the literature.

\section{LOSSLESS EXCITABILITY FOR PROPAGATING MODES}

In lossless waveguides, eigensolutions for which the wavenumber is pure real, pure imaginary and fully complex represent propagating waves, evanescent waves and inhomogeneous waves (decaying but oscillatory) respectively. Note that this section is focused on propagating modes in lossless waveguides.

Based on the SAFE method, a matrix expression of the excitability is proposed. This expression is shown to be the counterpart of the excitability tensor found in the literature by analytical approaches.

\section{A. Properties of propagating modes in lossless waveguides}

For lossless waveguides, the matrix of material properties $\mathbf{C}$ is real so that $\mathbf{K}_{1}, \mathbf{K}_{2}, \mathbf{K}_{3}$ and $\mathbf{M}$ are also real. $\mathbf{A}$ and $\mathbf{B}$ are thus hermitian: $\mathbf{A}^{* T}=\mathbf{A}$ and $\mathbf{B}^{* T}=\mathbf{B}(*$ denotes the complex conjugate). Besides, for propagating modes, wavenumbers are purely real and $k_{m}=k_{m}^{*}$. Then, taking the transpose conjugate of Eq. (9), it is straightforward to prove that the left eigenvectors are related to the right ones by:

$$
\hat{\mathbf{V}}_{m}=\hat{\mathbf{U}}_{m}^{*}
$$


This property has been used in Ref. [31] for analyzing the elastodynamic response of anisotropic plates.

The general biorthogonality relations (10) can now be rewritten in an interesting manner. From Eqs. (6), (7) and (15), the development of the expression $\hat{\mathbf{V}}_{n}^{T} \mathbf{B} \hat{\mathbf{U}}_{m}=$ $b_{m} \delta_{m n}$ yields:

$$
\mathbf{U}_{n}^{* T}\left(\mathbf{K}_{1}-\omega^{2} \mathbf{M}\right) \mathbf{U}_{m}-k_{n} k_{m} \mathbf{U}_{n}^{* T} \mathbf{K}_{3} \mathbf{U}_{m}=b_{m} \delta_{m n}
$$

Thanks to Eq. (4) written for mode $m$ (with $\tilde{\mathbf{F}}=\mathbf{0}$ ), one has $\left(\mathbf{K}_{1}-\omega^{2} \mathbf{M}\right) \mathbf{U}_{m}=-i k_{m}\left(\mathbf{K}_{2}-\mathbf{K}_{2}^{T}\right) \mathbf{U}_{m}-k_{m}^{2} \mathbf{K}_{3} \mathbf{U}_{m}$. Then after rearrangement, Eq. (16) can be rewritten as:

$i k_{m} \mathbf{U}_{n}^{* T}\left(\mathbf{K}_{2}^{T}+i k_{m} \mathbf{K}_{3}\right) \mathbf{U}_{m}-i k_{m} \mathbf{U}_{n}^{* T}\left(\mathbf{K}_{2}-i k_{n} \mathbf{K}_{3}\right) \mathbf{U}_{m}=b_{m} \delta_{m}$

Finally using Eq. (14), the biorthogonality relation becomes:

$$
\frac{i \omega}{4}\left(\mathbf{U}_{m}^{T} \mathbf{F}_{n}^{*}-\mathbf{U}_{n}^{* T} \mathbf{F}_{m}\right)=P_{m m} \delta_{m n}
$$

with the notation (justified later):

$$
P_{m m}=-\omega b_{m} / 4 k_{m}
$$

\section{B. Excitability matrix}

Using Eq. (15) and (19), the expansion (12) can be rewritten as:

$$
\mathbf{U}=\sum_{m=1}^{M} \mathbf{E}_{m} \tilde{\mathbf{F}}\left(k_{m}\right) e^{i k_{m} z}
$$

where:

$$
\mathbf{E}_{m}=\frac{i \omega}{4 P_{m m}} \mathbf{U}_{m} \mathbf{U}_{m}^{* T}
$$

$\mathbf{E}_{m}$ is a $N$ by $N$ matrix, $N$ denoting the number of dofs involved in the system (4).

In the above expansion, the contribution of nonpropagating modes has been neglected ( $M$ should be here understood as the number of propagating modes, excluding non-propagating). Hence, the expansion (20) together with (21) constitutes a far-field approximation, not applicable in the near-field region where nonpropagating modes may have a significant contribution in the excited field.

It can be seen from Eq. (20) that $\left(\mathbf{E}_{m}\right)_{i j}$ represents the displacement amplitude of the single wavemode $m$ at dof $i$ when a unit force acts at dof $j$. In particular, diagonal terms are ratios of modal displacement to applied force when both quantities are measured at the same location and direction in the cross-section. As a consequence, the matrix $\mathbf{E}_{m}$ can be viewed as the finite element version of the excitability function given in Refs. $[9,10] . \mathbf{E}_{m}$ will be referred to as the excitability matrix in the remainder.

\section{Interpretation based on analytical Auld's approach}

With the analytical approach of Auld ${ }^{6,8}$, the starting point for obtaining the modal expansion of the excited field is the reciprocity relation associated with the equations of elastodynamics. Details can be found elsewhere and calculations yield the following final result:

$$
\mathbf{u}(\mathbf{x}, z)=\sum_{m=1}^{M} \mathbf{e}_{m}(\mathbf{x}) \cdot \tilde{\mathbf{f}}\left(k_{m}\right) e^{i k_{m} z}
$$

where the applied force $\tilde{\mathbf{f}}$ is supposed to be concentrated at point $\mathbf{x}_{s}$ in the cross-section. In the above expression, $\tilde{\mathbf{f}}$ should be understood as $\tilde{\mathbf{t}}$ when the excitation source is a traction vector applied on the circumference $\delta S$ of the waveguide. $\mathbf{e}_{m}$ denotes the excitability tensor. Under the assumption of propagating modes in lossless waveguides, iqus expression is ${ }^{8,11}$.

$$
\mathbf{e}_{m}(\mathbf{x})=\frac{i \omega}{4 P_{m m}} \mathbf{u}_{m}(\mathbf{x}) \otimes \mathbf{u}_{m}^{*}\left(\mathbf{x}_{s}\right)
$$

where $\mathbf{x}=(x, y)$ denotes transverse variables (in the cross-section). $\mathbf{x}_{s}=\left(x_{s}, y_{s}\right)$ denotes the source position.

It can be observed that Eqs. (20) and (21) are the numerical counterparts of Eqs. (22) and (23). The reason of this direct analogy is explained below.

Indeed, the fundamental property that is used to derive Eq. (23) is the so-called Auld's complex orthogonality relation for propagating modes in lossless waveguides ${ }^{6}$, written as:

$$
P_{m n}=\frac{i \omega}{4} \int_{S}\left(\mathbf{u}_{m} \cdot \mathbf{t}_{n}^{*}-\mathbf{u}_{n}^{*} \cdot \mathbf{t}_{m}\right) d S=0 \text { if } m \neq n
$$

Such a relation indeed derives from the elastodynamic reciprocity theorem ${ }^{32}$.

With the SAFE approach, the fundamental property used to obtain Eq. (21) is the discrete biorthogonality relation (10). There is no obvious link between relations (10) and (24). However, in the particular case of propagating modes in lossless waveguides, one has shown that Eq. (10) can be rewritten as Eq. (18). Recalling the eigenforce definition (13), it is straightforward to see that the SAFE relation (18) is in fact the finite element form of Auld's complex orthogonality relation.

As a consequence, if one restricts the analysis to propagating modes in lossless waveguides, the procedure standardly followed with the SAFE method is in fact quite analogous to Auld's analytical approach.

Note that Eq. (24) is a biorthogonality relation in the sense that both displacement and stress components are involved. From a physical point of view, $P_{m m}$ is equal to the averaged power flow ${ }^{6}$ (cross-section and time averaged). $P_{m m}$ vanishes for non-propagating modes (evanescent or inhomogeneous), so that Eq. (23) is obviously not applicable for cut-off modes.

In the remainder of this paper, the expressions (21) or (23) will be referred to as the lossless excitability.

\section{GENERALIZED EXCITABILITY}

The SAFE expression given by Eq. (12) is general and not restricted to propagating modes in lossless waveguides. Starting from that result, this section proposes a generalized expression of the excitability, valid for nonpropagating modes and lossy waveguides. 


\section{A. General properties of the eigenproblem}

From a computational point of view, the general response given by Eq. (12) has the drawback of requiring the computation of both right and left eigenvectors. From a physical point of view, it is difficult to have a clear interpretation of left eigenvectors.

For isotropic materials, matrices $\mathbf{A}$ and $\mathbf{B}$ can be symmetrized by replacing the axial displacement $u_{z}$ with $i u_{z}$ - as done in Refs. [20, 21] - so that left and right eigenvectors become equal. In this paper, a simple relation between eigenvectors is obtained in the general case of anisotropic materials.

First, one recalls that if $k_{m}$ is an eigenvalue of the quadratic formulation (4), then $-k_{m}$ is also an eigenvalue. This is due to the symmetry of $\mathbf{K}_{1}, \mathbf{K}_{3}$ and $\mathbf{M}$ and the property $\operatorname{det} \mathbf{D}^{T}=\operatorname{det} \mathbf{D}(\mathbf{D}$ is any matrix). Hence, the eigenproblem has two sets of eigensolutions $\left(k_{m}^{+}, \mathbf{U}_{m}^{+}\right)$ and $\left(k_{m}^{-}, \mathbf{U}_{m}^{-}\right)$, where $k_{m}^{-}=-k_{m}^{+}(m=1, \ldots, M)$, representing $M$ positive-going and $M$ negative-going wave modes.

Let us redenote $\left(-k_{m}, \mathbf{U}_{-m}\right)$ the opposite-going mode associated with the eigensolution $\left(k_{m}, \mathbf{U}_{m}\right)$. From Eq. (9) and the symmetry of $\mathbf{B}$, the left eigenvector $\hat{\mathbf{V}}_{m}$ of mode $m$, associated with the eigenvalue $k_{m}$, satisfies:

$$
\left\{\mathbf{A}^{T}-k_{m} \mathbf{B}\right\} \hat{\mathbf{V}}_{m}=\mathbf{0}
$$

Then, it can be readily checked that the above eigenvalue problem is satisfied by the following vector:

$$
\hat{\mathbf{V}}_{m}=\left\{\begin{array}{c}
\mathbf{U}_{-m} \\
k_{m} \mathbf{U}_{-m}
\end{array}\right\}
$$

which allows to get the left eigenvectors directly from the right ones (i.e. the guided modes). Note that thanks to this expression, solving the left eigenproblem is not needed.

Following the previous section, let us develop the general biorthogonality relation $\hat{\mathbf{V}}_{n}^{T} \mathbf{B} \hat{\mathbf{U}}_{m}=b_{m} \delta_{m n}$. Using Eqs. (14) and (26), one finally gets after rearrangement:

$$
\frac{i \omega}{4}\left(\mathbf{U}_{m}^{T} \mathbf{F}_{-n}-\mathbf{U}_{-n}^{T} \mathbf{F}_{m}\right)=Q_{m,-m} \delta_{m n}
$$

with the notation:

$$
Q_{m,-m}=-\omega b_{m} / 4 k_{m}
$$

One emphasizes that no specific assumption has been made. In particular, $\mathbf{C}$ can be complex, as in the viscoelastic case. Hence, properties (26) and (27) are general and still hold for non-propagating modes and lossy waveguides.

\section{B. Generalized excitability matrix}

Using Eq. (26), one has: $\hat{\mathbf{V}}_{m}^{T} \hat{\mathbf{F}}=k_{m} \mathbf{U}_{-m}^{T} \mathbf{F}$. With notation (28), the expansion (12) can still be rewritten in the form of Eq. (20), but with the new modal excitability matrix:

$$
\mathbf{E}_{m}=\frac{i \omega}{4 Q_{m,-m}} \mathbf{U}_{m} \mathbf{U}_{-m}^{T}
$$

The interpretation of $\left(\mathbf{E}_{m}\right)_{i j}$ does not change from the previous section. Yet, the above expression is general and not restricted to propagating modes in lossless waveguides. The expansion (20) together with (29) is valid in the far-field as well as in the near-field region ( $z$ must yet lie outside the source region, as recalled in Sec. II).

The excitability defined by Eq. (29) will be refered to as the generalized excitability matrix in the remainder.

For propagating modes in lossless waveguides, Eqs. (15), (26) and (14) allow to get the following equalities:

$$
\mathbf{U}_{-m}=\mathbf{U}_{m}^{*}, \quad \mathbf{F}_{-m}=\mathbf{F}_{m}^{*}
$$

From Eq. (27), one has $Q_{m,-m}=P_{m m}$, and the generalized excitability (29) reduces to the lossless excitability (21).

\section{Link with an analytical approach}

Provided the straightforward analogy established between the analytical and numerical approaches in Sec. III.C, it can be deduced from Eq. (29) that the analytical expression of the generalized excitability is:

$$
\mathbf{e}_{m}(\mathbf{x})=\frac{i \omega}{4 Q_{m,-m}} \mathbf{u}_{m}(\mathbf{x}) \otimes \mathbf{u}_{-m}\left(\mathbf{x}_{s}\right)
$$

and the analytical form associated with the numerical biorthogonality relation (27) is:

$$
Q_{m,-n}=\frac{i \omega}{4} \int_{S}\left(\mathbf{u}_{m} \cdot \mathbf{t}_{-n}-\mathbf{u}_{-n} \cdot \mathbf{t}_{m}\right) d S=0 \text { if } m \neq n
$$

The relation (32) is encountered in the literature, under the following slightly different form: $Q_{m, n}=0$ if $m \neq-n$, which is usually referred to as the real biorthogonality relation ${ }^{6}$. Unlike the averaged power flow $P_{m m}$, note that the normalization factor $Q_{m,-m}$ remains nonzero for all modes including non-propagating ones ${ }^{33}$.

In fact, the analytical derivation of Eq. (31) can be obtained from the real reciprocity relation of Auld ${ }^{6}$ (instead of the complex relation). It could be checked that writing the real reciprocity expression for two different wave fields, one corresponding to a given $-m$ mode and the other to the excited field, and applying the real biorthogonality relation (32) yields the generalized excitability result given by Eq. (31).

Surprisingly, the real orthogonality relation has been considered in scattering analysis ${ }^{6,33,34}$, but not for waveguide excitation problems for which the complex orthogonality relation has generally been preferred ${ }^{6,8,11,14,15}$.

\section{Green's matrix}

The Green's matrix can be conveniently expressed in terms of modal excitabilities.

By definition, the response $\mathbf{u}(\mathbf{x}, z)$ is given by the convolution product between the Green's tensor $\mathbf{G}(\mathbf{x}, z)$ and the excitation $\mathbf{f}(z)$ in the $(z, \omega)$ domain, or equivalently 
in the wavenumber domain: $\tilde{\mathbf{u}}(\mathbf{x}, k)=\tilde{\mathbf{G}}(\mathbf{x}, k) \cdot \tilde{\mathbf{f}}(k)$. Applying the inverse spatial Fourier transform and the Cauchy residue theorem, one has ${ }^{5,35}$ :

$$
\mathbf{u}(\mathbf{x}, z)=\sum_{m=1}^{M} i \operatorname{Res}\left(\tilde{\mathbf{G}}(\mathbf{x}, k) ; k=k_{m}\right) \cdot \tilde{\mathbf{f}}\left(k_{m}\right) e^{i k_{m} z}
$$

where $\operatorname{Res}(f ; k)$ denotes the residue of $f$ at $k$. Identifying the above expression with Eq. (22), one has the equality: $i \operatorname{Res}\left(\tilde{\mathbf{G}}(\mathbf{x}, k) ; k=k_{m}\right)=\mathbf{e}_{m}(\mathbf{x})$.

The inverse spatial Fourier transform of $\tilde{\mathbf{G}}(\mathbf{x}, k)$ yields after application of the Cauchy residue theorem: $\mathbf{G}(\mathbf{x}, z)=\sum_{m=1}^{M} i \operatorname{Res}\left(\tilde{\mathbf{G}}(\mathbf{x}, k) ; k=k_{m}\right) e^{i k_{m} z}$.

Hence, the expression of Green's tensor can be expressed in terms of the excitability, as follows ${ }^{11}$ :

$$
\mathbf{G}(\mathbf{x}, z)=\sum_{m=1}^{M} \mathbf{e}_{m}(\mathbf{x}) e^{i k_{m} z}
$$

\section{RESULTS}

For three test cases, this section briefly compares results obtained from the lossless excitability (21) with the generalized one (29). As already mentioned in Sec. I, the excitability in lossy waveguides is sometimes approximated with the lossless expression. The error made from the standard formula approximation is highlighted. For simplicity, the examples are two-dimensional and corresponds to layered plates excited by line sources. For clarity, $\mathbf{L}_{x y}=\mathbf{L}_{x}$ and $\mathbf{L}_{z}$ are the operators of two-dimensional plane strain given by:

$$
\mathbf{L}_{x}=\left[\begin{array}{cc}
\partial / \partial x & 0 \\
0 & 0 \\
0 & \partial / \partial x
\end{array}\right], \mathbf{L}_{z}=\left[\begin{array}{ll}
0 & 0 \\
0 & 1 \\
1 & 0
\end{array}\right]
$$

Throughout this section, the excitabilities will be computed from displacement-force ratios measured at the free surface in the normal direction (i.e. $x$-direction). The SAFE method will be employed. One will also present the lossless excitability calculated analytically from the software Disperse ${ }^{36}$ in order to check the accuracy of numerical models.

\section{A. Viscoelastic waveguide}

This first test case compares the excitabilities calculated for a bilayered plate, with and without viscoelasticity. This test case is taken from the work of Simonetti ${ }^{37}$. The relevant material parameters are summarised in Table I.

First, let us consider the non-viscoelastic case. Figure 1 gives the SAFE dispersion curves in phase velocity for two modes of interest, denoted $M_{0}$ and $M_{1}$, in agreement with the results calculated in Ref. [37] from the software Disperse. Figure 2 shows the SAFE modal excitabilities, as well as Disperse lossless excitability. As
TABLE I: Characteristics of the bilayered plate

\begin{tabular}{c|c|c|c|c}
\hline \hline & $c_{s}(\mathrm{~m} / \mathrm{s})$ & $c_{l}(\mathrm{~m} / \mathrm{s})$ & $\rho\left(\mathrm{kg} / \mathrm{m}^{3}\right)$ & thickness $(\mathrm{mm})$ \\
\hline Layer 1 (elastic) & 900 & 1700 & 1250 & 9 \\
Layer 2 (metallic) & 3260 & 5960 & 7930 & 8 \\
\hline \hline
\end{tabular}

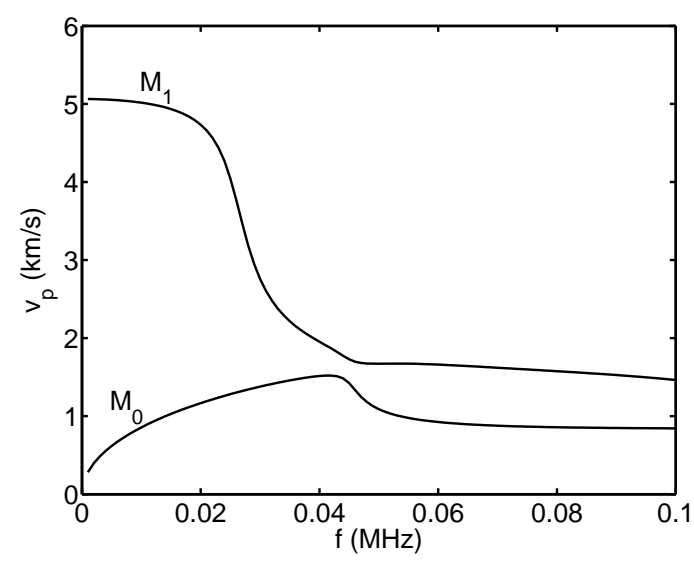

FIG. 1: Dispersion curves in phase velocity for modes $M_{0}$ and $M_{1}$ computed from the SAFE method.

expected, the lossless excitabilities are equal to the generalized ones because both $M_{0}$ and $M_{1}$ modes are propagating modes in a lossless waveguide.

Next, let us consider the viscoelastic case when a large material attenuation is considered. The metallic layer is still lossless but $\alpha_{s}=\alpha_{l}=1$ is set for the viscoelastic layer (Layer 1), $\alpha_{s}$ and $\alpha_{l}$ denoting the shear and longitudinal bulk wave attenuations (in Neper per wavelength).

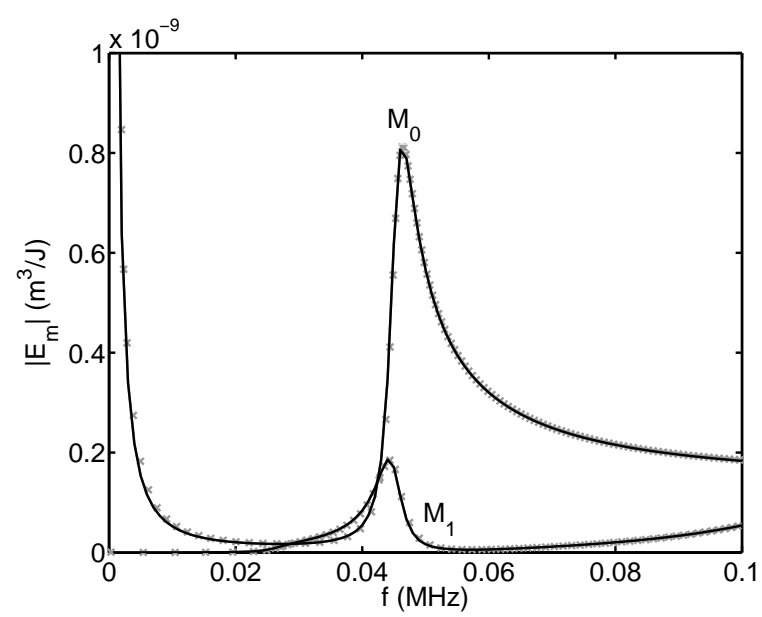

FIG. 2: $M_{0}$ and $M_{1}$ excitabilities computed from the SAFE method (black) and calculated from Disperse (crosses). 

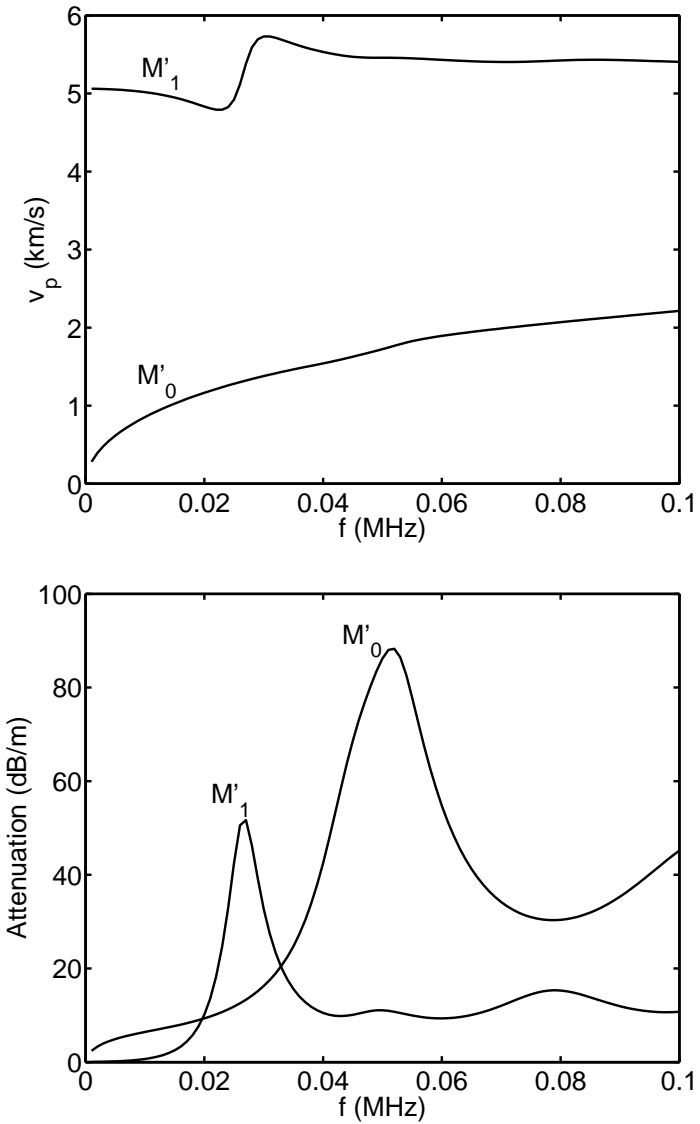

FIG. 3: Dispersion curves in phase velocity (top) and attenuation (bottom) for modes $M_{0}^{\prime}$ and $M_{1}^{\prime}$ computed from the SAFE method.

Complex bulk wave velocities are defined from:

$$
\tilde{c}_{s, l}=\frac{c_{s}}{1+\mathrm{i} \frac{\alpha_{s, l}}{2 \pi}}
$$

The matrix of elasticity coefficients $\mathbf{C}$ becomes complex. The lossless excitability expression is not valid in that case and becomes approximate.

Figure 3 shows the SAFE dispersion curves in phase velocity and attenuation for the first two less attenuated modes, denoted $M_{0}^{\prime}$ and $M_{1}^{\prime}$ (also in agreement with Ref. [37]).

Figure 4 exhibits the modal lossless and generalized excitabilities. In this example, it is remarkable that although the material attenuation is high and the viscoelasticity strongly modifies velocity curve patterns (compare Figs. 1 and 3), the lossless excitability still yields good modulus approximation.

For a given mode, it can be observed that the largest amplitude difference between the lossless and the generalized formula occurs at a frequency for which the modal attenuation is maximum (roughly $0.05 \mathrm{MHz}$ for $M_{0}^{\prime}, 0.025 \mathrm{MHz}$ for $M_{1}^{\prime}$ ): as expected, the deviation of the approximate lossless excitability is increasing with loss.

As shown in Fig. 4 (bottom), the phase strongly dif-
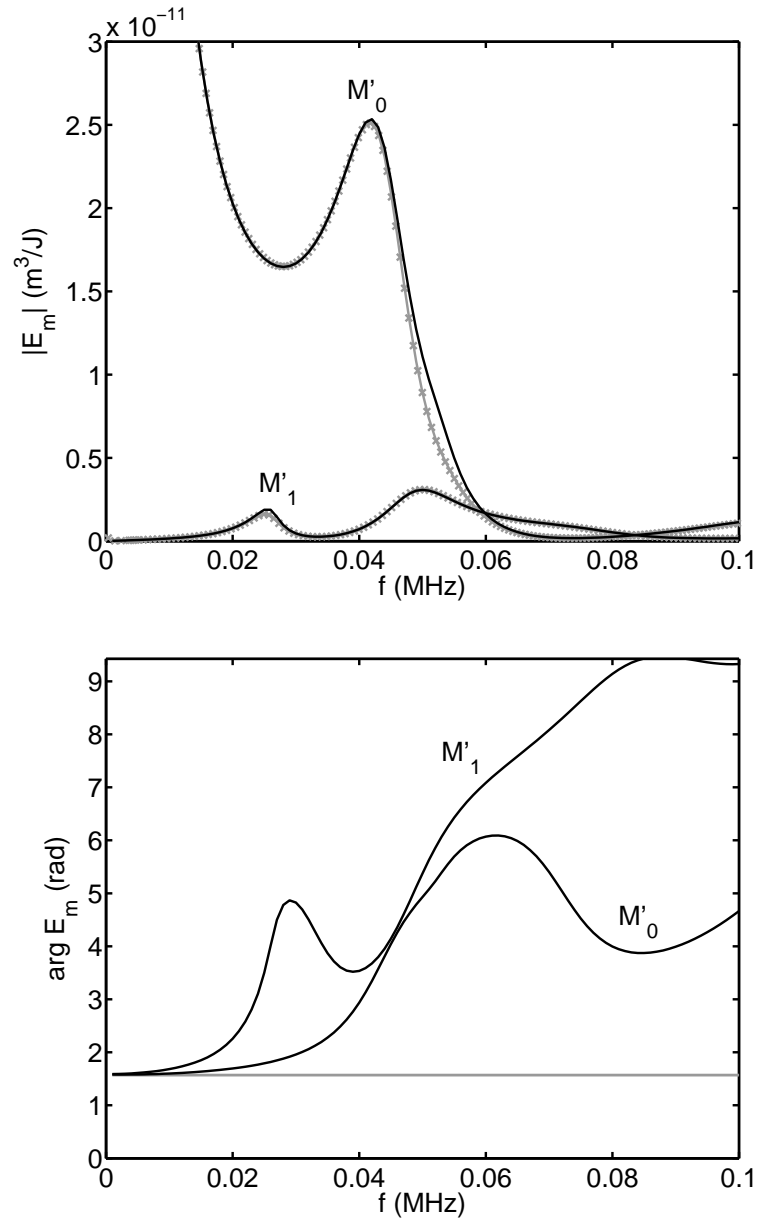

FIG. 4: $M_{0}^{\prime}$ and $M_{1}^{\prime}$ excitabilities, lossless (gray) and generalized (black), in modulus (top) and phase

(bottom) computed from the SAFE method. Crosses: lossless excitability calculated from Disperse. Horizontal gray line at $+\pi / 2$ : phase of lossless excitability.

fers. The generalized excitability is fully complex, with frequency varying phase. The lossless excitability remains constant and pure imaginary (the diagonal terms of Eq. (21) are always pure positive imaginary), yielding a constant $+\pi / 2$ phase.

\section{B. Excitation of non-propagating modes}

This second test deals with a numerical experiment simulating a zero group velocity (ZGV) resonance. A $\mathrm{ZGV}$ resonance is a local resonance occurring at a frequency of Lamb modes where the group velocity vanishes while the phase velocity remains non-zero ${ }^{38-40}$. Below the ZGV frequency, the modes contributing to the resonance are cut-off. Hence, the contribution of nonpropagating modes is expected to be significant.

A steel plate of thickness $a=1 \mathrm{~cm}$ is considered, with Young modulus $E=210 \mathrm{GPa}$, Poisson ratio $\nu=0.3$ and density $\rho=7800 \mathrm{~kg} / \mathrm{m}^{3}$. The first ZGV frequency can be obtained from the empirical formula ${ }^{41,42}: f_{0}=\beta c_{l} / 2 a \simeq$ 

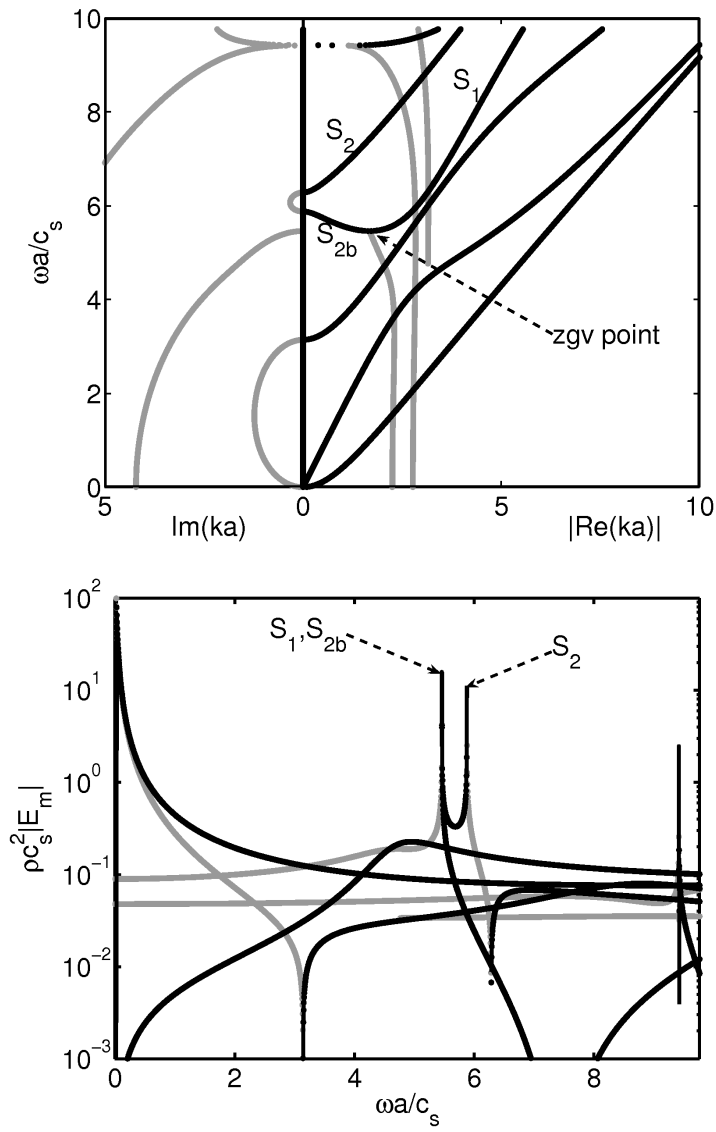

FIG. 5: Dimensionless dispersion curves (top) and excitabilities (bottom) computed from the SAFE method. Black: propagating modes, gray: non-propagating modes. Disperse lossless excitabilities are superimposed to black lines (excluding non-propagating modes).

$280 \mathrm{kHz}$, where $\beta$ is a correction factor that depends on $\nu$ ( $\beta \simeq 0.93$ for $\nu=0.3$ - see Ref. [42]). As already proved from theory and experiment ${ }^{38,42}$, the resonance is caused by the interference between the two symmetric $S_{1}$ and $S_{2 b}$ modes propagating with opposite phase velocities and generated with comparable amplitudes (the subscript $b$ means that $S_{2 b}$ is a backward mode near the resonance).

Figure 5 (top) shows the dimensionless wavenumberfrequency dispersion curves computed from the SAFE method. The first $\mathrm{ZGV}$ resonance is observed at the zero slope point of $S_{1}$ and $S_{2 b}$ curves $\left(k_{0} a, \omega_{0} a / c_{s}\right)=$ $(1.68,5.47)$, giving a dimensional frequency of $280 \mathrm{kHz}$ in agreement with the empirical formula.

Near the resonance, the contribution of $S_{1}$ and $S_{2}$ modes is expected to be significantly greater than the other modes ${ }^{41,42}$. This is confirmed by looking at the excitabilities shown in Fig. 5 (bottom).

Let us recall that lossless excitabilities curves cannot be calculated for non-propagating modes (due to zero power flow), and stop at cut-off frequencies. Such curves hence coincide with black lines in the figure (excluding gray ones).

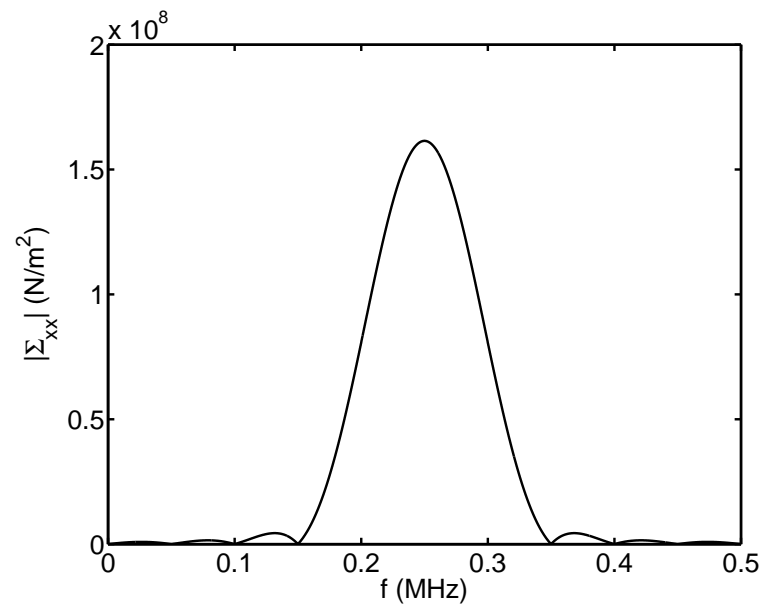

FIG. 6: Frequency spectrum of the excitation.

Now, the plate is excited by a line force (along $y$ ) located at the surface $x=0$ and oriented along the $x$ direction (normal to the plate). The time excitation is a Hanning-windowed 5 cycles sinusoidal toneburst centred at $250 \mathrm{kHz}$, close to the first ZGV frequency. Its spectrum is given by Fig. 6 .

Figure 7 gives the response spectrum for the $x$ displacement computed with the SAFE approach from Eq. (20), with the lossless excitability (21) as well as the generalized one (29), at a distance close to the source $z=5 \mathrm{~mm}$. In order to avoid interfence nodes ${ }^{38,42}$, this distance has been chosen as less than a quarter wavelength of the $S_{1}$ and $S_{2 b}$ modes $\left(\lambda=2 \pi / k_{0}=3.74 \mathrm{~cm}\right)$. In the modal expansions, one has computed and kept the less attenuated modes satisfying $|\operatorname{Im}(k)| \leqslant 1 \mathrm{~mm}^{-1}$. This ensures that the contribution of higher order modes is negligible at the position $z=5 \mathrm{~mm}$ (less than $e^{-1 \times 5} \simeq$ 0.007).

As expected, the maximum response occurs at the $\mathrm{ZGV}$ frequency $f_{0}$. Although the spectrum seems to be almost identical between the lossless and the generalized formula, a zoom in the range $[260-320] \mathrm{kHz}$ reveals differences - see Fig. 7 (bottom). Between 280 and $300 \mathrm{kHz}$, the $S_{1}$ and $S_{2 b}$ modes are both propagating and the lossless excitability gives exactly the same results as the generalized one. Yet, both modes are inhomogeneous below $280 \mathrm{kHz}$ and the $S_{2 b}$ mode is evanescent in a narrow frequency range starting from $300 \mathrm{kHz}$ (i.e. $\omega a / c_{s} \simeq 5.86$ in Fig. 5 (top)). Then the lossless excitability, not applicable to non-propagating modes, gives erroneous abrupt changes near the cut-off frequencies $280 \mathrm{kHz}$ and $300 \mathrm{kHz}$.

Figure 8 (top) gives the time signal obtained from the inverse Fourier transform of the generalized formula, up to time $t=5 \mathrm{~ms}$. One can observe the typical behavior of a zero-group velocity wave, oscillating with a very slow exponential decay ${ }^{38}$. As shown by Fig. 8 (bottom), this phenomenon cannot be recovered with the lossless formula. Indeed, neglecting the contribution of nonpropagating modes acts as non-causal modal filters (highor band-pass), yielding erroneous time waveforms (arti- 

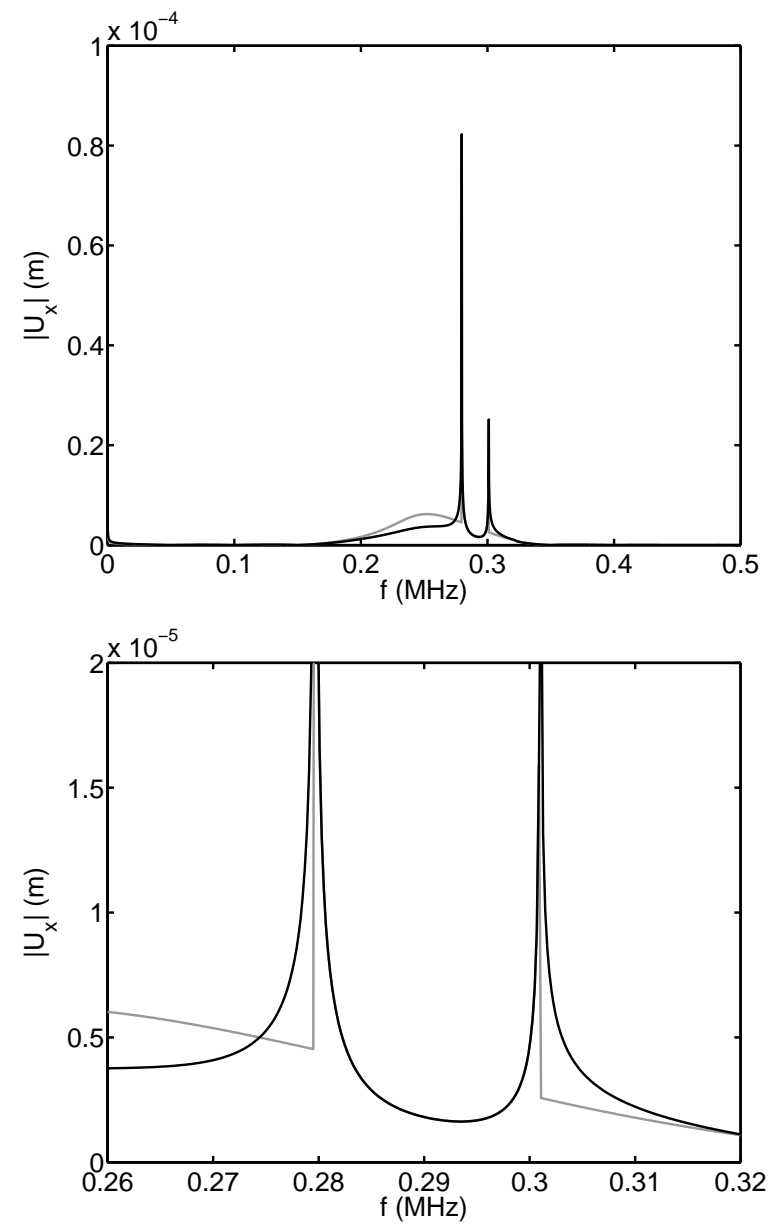

FIG. 7: Top: SAFE computation of the frequency spectrum of the $x$-displacement response obtained from the generalized excitability (black lines) and from the lossless excitability (gray lines). Bottom: zoom between 260 and $320 \mathrm{kHz}$.

ficial growth at the end of the signal in the figure).

As already mentioned in Sec. II.C, one recalls that only modes travelling in the proper direction (here, the positive direction) must be selected into the modal expansion. It has to be noticed that Fig. 5 (top) actually shows the dispersion curves of every mode included in the expansion (positive-going modes). The negative slope observed for the backward mode $\left(S_{2 b}\right)$ is due to the fact that the figure plots the absolute value of $\operatorname{Re}(k a)$ : dropping the absolute value, the slope becomes properly positive because the wavenumber of a positive-going backward mode have a negative real part.

\section{Excitation of a layered half-space}

In this last test case, a layered half space made of three layers is considered with characteristics given by Table II. This example is taken from Ref. [16] and represents a pavement construction. Layer 3 is a halfspace, soft compared to the first two layers. No material attenuation is
TABLE II: Characteristics of the three-layer model

\begin{tabular}{l|c|c|c|c}
\hline \hline & $c_{s}(\mathrm{~m} / \mathrm{s})$ & $c_{l}(\mathrm{~m} / \mathrm{s})$ & $\rho\left(\mathrm{kg} / \mathrm{m}^{3}\right)$ & thickness $(\mathrm{m})$ \\
\hline Layer 1 & 1400 & 2914 & 2000 & 0.2 \\
Layer 2 & 500 & 1041 & 2000 & 0.6 \\
Layer 3 & 100 & 208 & 2000 & $\infty$ \\
\hline \hline
\end{tabular}

included in this model. Nevertheless, modes are attenuated in the axial direction $(\operatorname{Im}(k)>0)$ due to the loss of energy into the halfspace. Such modes are called leaky modes.

In the numerical SAFE model, the presence of a semiinfinite layer is simulated thanks to an absorbing layer, which consists in truncating the unbounded layer (Layer 3 ) and adding an artificial growing viscoelasticity to the material properties. The open problem is hence transformed into a closed one and the numerical approach proposed in the present paper can be applied without difficulty (the problem becomes similar to a closed viscoelastic waveguide).

Following Ref. [43], the law adopted for the absorbing layer is:

$$
C_{i j}^{\prime}=C_{i j}\left\{1-\mathrm{i} s\left(\frac{x-d}{h}\right)^{3}\right\}
$$

where $d$ and $h$ denotes the position and thickness of Layer 3 and $s$ is a user-defined parameter. In the simulation, one has set $h=0.6 \mathrm{~m}$. A parametric study has been conducted leading to $s=10$. Figure 9 shows the SAFE dispersion curves in phase velocity and attenuation. In order to validate the parameters of the absorbing layer, Disperse results are also shown for this test case. Good agreement is found.

Figure 10 compares the lossless and the generalized excitabilities calculated from the SAFE approach. As already mentioned in Sec. V.A, the error in modulus made with the lossless excitability is greater at frequencies of highest modal attenuation. In this example, these frequencies also coincide with highest excitabilities. The amplitudes of modes 3 and 6 are particularly affected at $500 \mathrm{~Hz}$ and $570 \mathrm{~Hz}$ respectively. While the excitability of mode 3 is higher with the generalized formula, the excitability of mode 6 becomes substantially lower. In particular, the highest excitability is actually given by mode 3 (instead of mode 6 with the lossless formula approximation).

Figure 10 (bottom) shows the phase of excitabilities. As already observed in Sec. V.A, the phase is strongly dependent on the modes and frequency (while the phase of the lossless excitability remains equal to $+\pi / 2$ ).

It could be argued that the results of Fig. 10 may be dependent of the parameters of the absorbing layers with the SAFE method. However, one has checked that increasing the thickness $h$ yields negligible differences.

Furthermore in Fig. 10 (top), the lossless excitability calculated with Disperse has also been plotted. From a theoretical point of view, the proof of orthogonality of leaky modes is still an open question (beyond the scope 

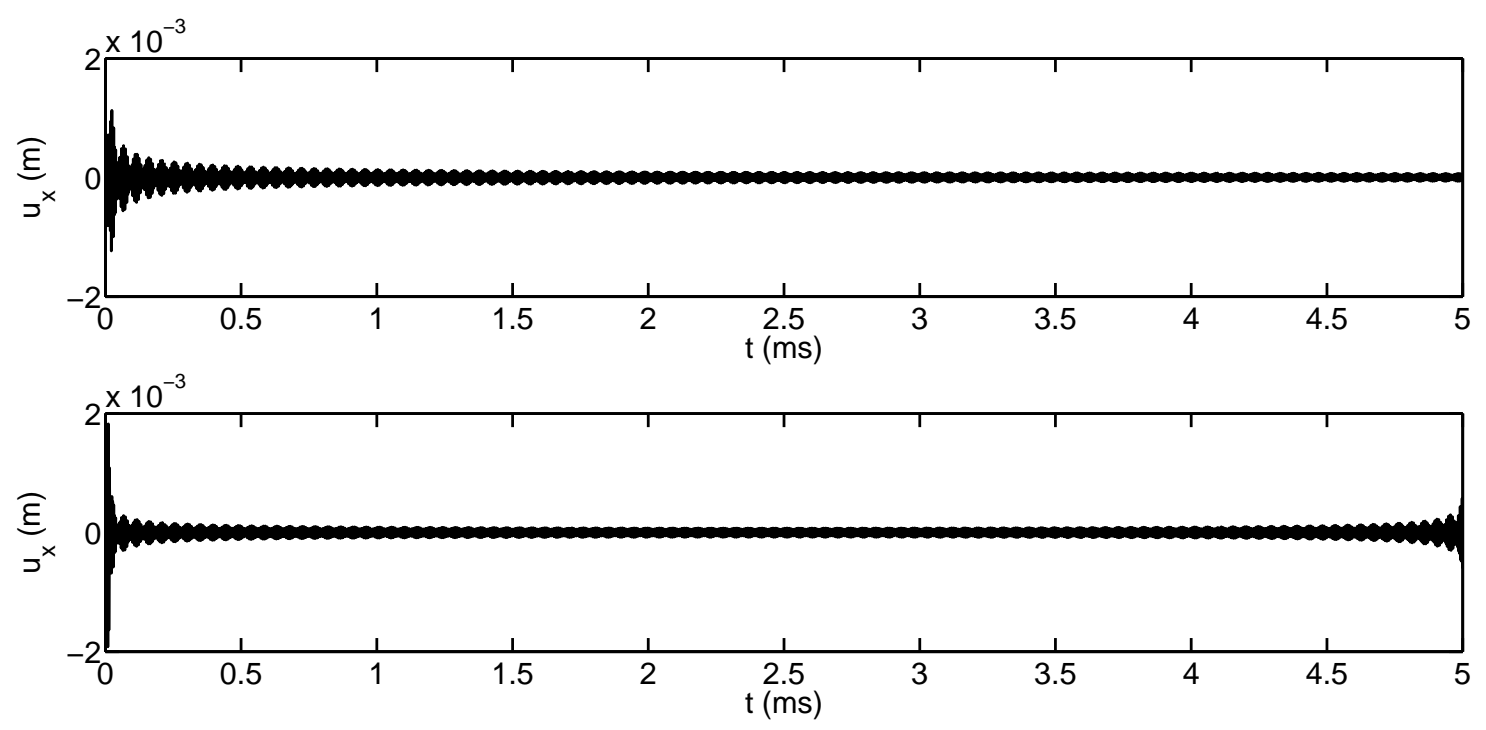

FIG. 8: SAFE $x$-displacement time response obtained from the generalized excitability (top) and the lossless excitability (bottom).
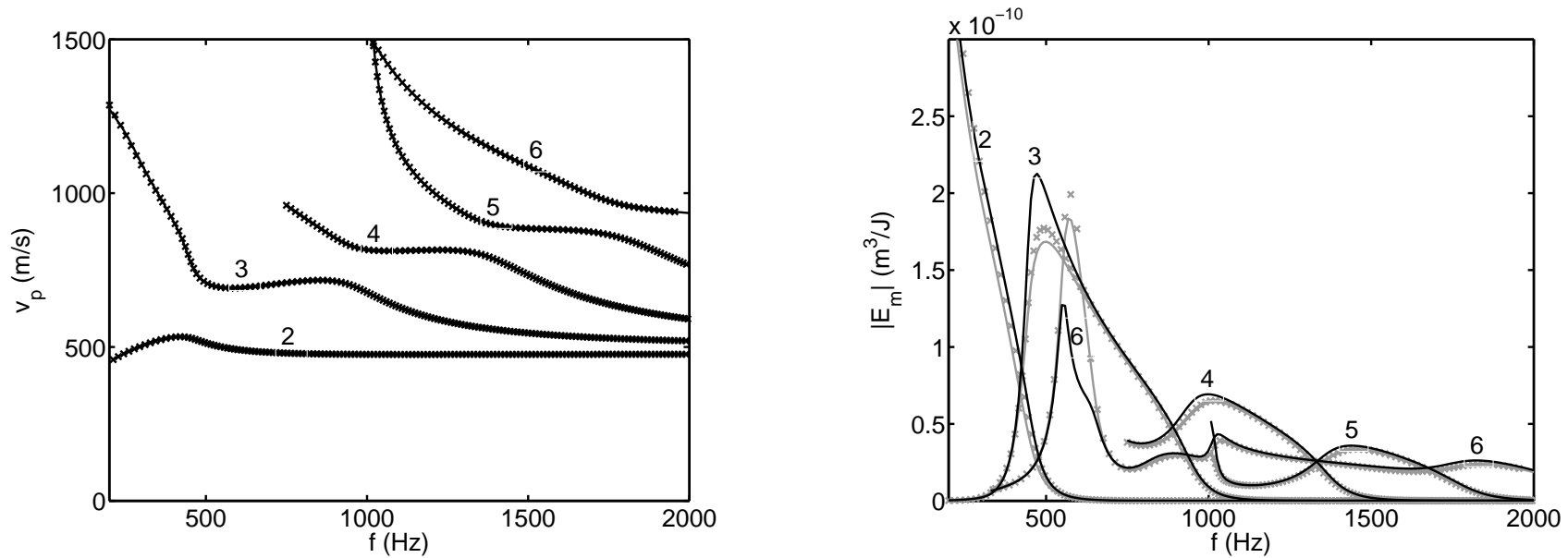

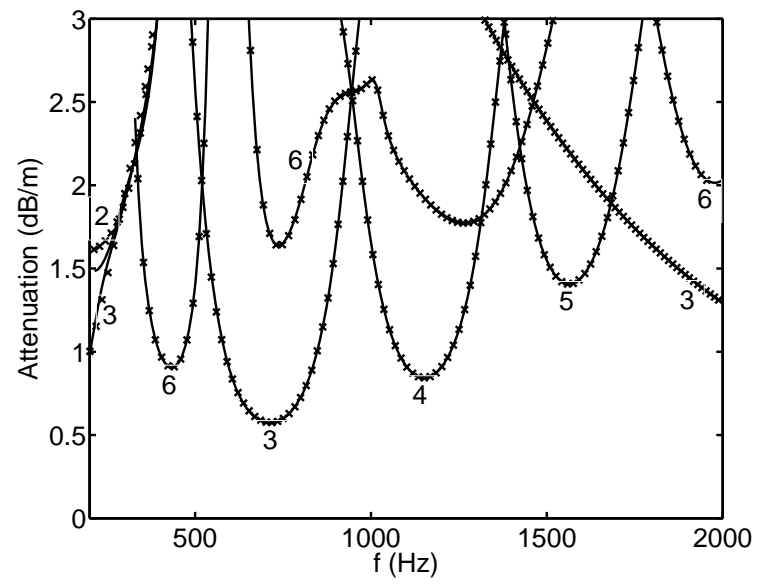

FIG. 9: Dispersion curves in phase velocity (top) and attenuation (bottom) computed from the SAFE method. Crosses: Disperse results.

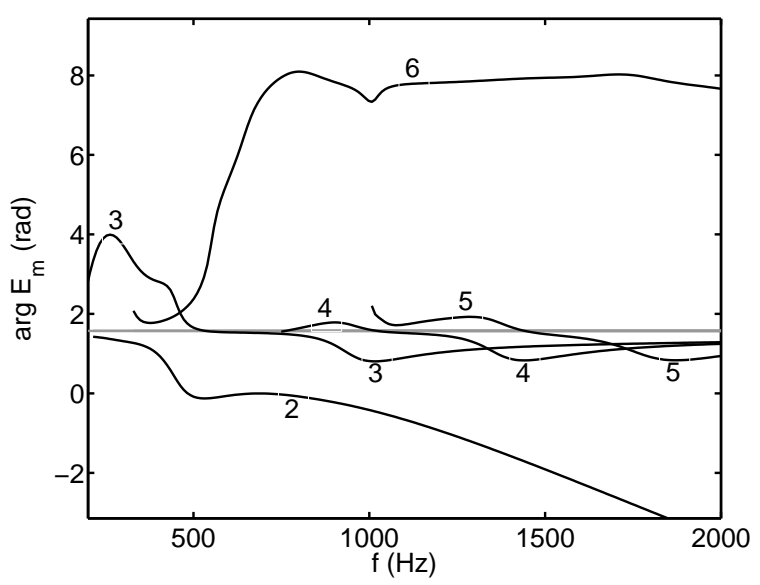

FIG. 10: Excitabilities in modulus (top) and phase (bottom). Same legend as Fig. 4. 
of this paper) because the wave fields of such modes do not vanish at infinity in the half-space. As a consequence, an orthogonality relation written with an integral including the whole half-space may be divergent, as noticed in Ref. [33]. To circumvent this problem, these authors have proposed to use the integral restricted over the finite area excluding the half-space layer and have checked that such an approximation yields small error in modal orthogonality. This explains why small discrepancies with the SAFE lossless excitabilities are observed in Fig. 10 (top) near the maxima of modes 3 and 6: the halfspace (i.e. Layer 3 ) is not included in the power integral of Disperse ${ }^{36}$. This has been checked by a numerical test consisting of keeping only the dofs of Layers 1 and 2 in the SAFE computation of the power flow given by Eq. (18), yielding the same results as Disperse (curves not shown for clarity of figure).

Finally, one must emphasize that open waveguides could be efficiently modeled by perfectly matched layers (PML), as recently proposed in Ref. [44]. With a PML method, SAFE matrices are complex but their symmetry is still preserved (as in the viscoelastic case) so that the numerical approach presented in this paper still applies.

\section{CONCLUSION}

A generalized expression has been proposed for the modal excitability of elastic waveguides. This expression remains applicable for lossy waveguides as well as nonpropagating modes. It has been derived for analytical and SAFE approaches. For both approaches, the proper orthogonality relation is shown to be a form of Auld's real orthogonality relation, involving opposite-going modes.

From a computational point of view, only the right eigensolutions are needed in the SAFE method. Solving the left eigenproblem is avoided for response computations.

Differences between the lossless and the generalized excitabilities have been highlighted for viscoelastic materials, near field and local phenomena for which the contribution of non-propagating modes is significant, as well as leaky modes in open waveguides. In these three cases, the lossless excitability might yield acceptable response approximations in modulus in the frequency domain, but not in phase leading to inaccurate time results.

1 P. D. Wilcox, M. J. S. Lowe, and P. Cawley, "Mode and transducer selection for long range Lamb wave inspection", Journal of Intelligent Material Systems and Structures 12, 553-565 (2001).

${ }^{2}$ I. A. Viktorov, Rayleigh and Lamb Waves (Plenum, New York) (1967), $154 \mathrm{p}$.

${ }^{3}$ W. A. Green and E. R. Green, "Elastic waves in fibre composite laminates", Ultrasonics 38, 228-231 (2000).

4 A. Cheng, T. W. Murray, and J. D. Achenbach, "Simulation of laser-generated ultrasonic waves in layered plates", Journal of the Acoustical Society of America 110, 29022913 (2001).

${ }^{5}$ E. V. Glushkov, N. V. Glushkova, W. Seemann, and O. V. Kvasha, "Elastic wave excitation in a layer by piezoceramic patch actuators", Acoustical Physics 52, 398-407 (2006).
${ }^{6}$ B. A. Auld, Acoustic Fields and Waves in Solids, volume II, 2nd edition (Krieger, Malabar, FL) (1990), 432 p.

7 J. J. Ditri and J. L. Rose, "Excitation of guided elastic wave modes in hollow cylinders by applied surface tractions", Journal of Applied Physics 72, 2589-2597 (1992).

${ }^{8}$ I. Nunez, R. K. Ing, C. Negreira, and M. Fink, "Transfer and green functions based on modal analysis for Lamb waves generation", Journal of the Acoustical Society of America 107, 2370-2378 (2000).

9 P. D. Wilcox, "Lamb wave inspection of large structures using permanently attached transducers", Ph.D. thesis, Imperial College of Science, Technology and Medecine, University of London (1998).

10 P. Wilcox, M. Evans, O. Diligent, and P. Cawley, "Dispersion and excitability of guided acoustic waves in isotropic beams with arbitrary cross-section", in Review of Progress in Quantitative NDE, edited by D. O. Thompson and D. E. Chimenti, volume 615, 203-210 (AIP Conference Proceedings, New York) (2002).

11 A. Velichko and P. D. Wilcox, "Modeling the excitation of guided waves in generally anisotropic multilayered media", Journal of the Acoustical Society of America 121, 60-69 (2007).

12 S. Guilbaud and B. Audoin, "Measurement of the stiffness coefficients of a viscoelastic composite material with laser-generated and detected ultrasound", Journal of the Acoustical Society of America 105, 2226-2235 (1999).

13 A. Mal, "Elastic waves from localized sources in composite laminates", International Journal of Solids and Structures 39, 5481-5494 (2002).

14 X. Jia, "Modal analysis of Lamb wave generation in elastic plates by liquid wedge transducers", Journal of the Acoustical Society of America 101, 834-841 (1997).

15 L. Duquenne, E. Moulin, J. Assaad, and S. Grondel, "Transient modeling of Lamb waves generated in viscoelastic materials by surface bonded piezoelectric transducers", Journal of the Acoustical Society of America 116, 133-141 (2004).

16 N. Ryden and M. J. S. Lowe, "Guided wave propagation in three-layer pavement structures", Journal of the Acoustical Society of America 116, 2902-2913 (2004).

17 L. Gavric, "Computation of propagative waves in free rail using a finite element technique", Journal of Sound and Vibration 185, 531-543 (1995).

18 T. Hayashi, W.-J. Song, and J. L. Rose, "Guided wave dispersion curves for a bar with an arbitrary cross-section, a rod and rail example", Ultrasonics 41, 175-183 (2003).

19 V. Damljanovic and R. L. Weaver, "Propagating and evanescent elastic waves in cylindrical waveguides of arbitrary cross-section", Journal of the Acoustical Society of America 115, 1572-1581 (2004).

20 V. Damljanovic and R. L. Weaver, "Forced response of a cylindrical waveguide with simulation of the wavenumber extraction problem", Journal of the Acoustical Society of America 115, 1582-1591 (2004).

${ }^{21} \mathrm{P}$. W. Loveday, "Simulation of piezoelectric excitation of guided waves using waveguide finite elements", IEEE Transactions on Ultrasonics, Ferroelectrics, and Frequency Control 55, 2038-2045 (2008).

22 I. Bartoli, A. Marzani, F. L. di Scalea, and E. Viola, "Modeling wave propagation in damped waveguides of arbitrary cross-section", Journal of Sound and Vibration 295, 685707 (2006).

23 A. Marzani, "Time-transient response for ultrasonic guided waves propagating in damped cylinders", International 
Journal of Solids and Structures 45, 6347-6368 (2008).

${ }^{24}$ G. R. Liu and J. D. Achenbach, "Strip element method to analyze wave scattering by cracks in anisotropic laminated plates", Journal of Applied Mechanics 62, 607-613 (1995).

25 J. Zhu and A. H. Shah, "A hybrid method for transient wave scattering by flaws in composite plates", International Journal of Solids and Structures 34, 1719-1734 (1997).

${ }^{26}$ F. Tisseur and K. Meerbergen, "The quadratic eigenvalue problem", SIAM Review 43, 235-286 (2001).

27 S. Adhikari and M. I. Friswell, "Eigenderivative analysis of asymmetric non-conservative systems", International Journal for Numerical Methods in Engineering 51, 709-733 (2001).

28 J. M. Renno and B. R. Mace, "On the forced response of waveguides using the wave and finite element method", Journal of Sound and Vibration 329, 5474-5488 (2010).

${ }^{29}$ F. Treyssede, "Mode propagation in curved waveguides and scattering by inhomogeneities: Application to the elastodynamics of helical structures", Journal of the Acoustical Society of America 129, 1857-1868 (2011).

30 F. Benmeddour, F. Treyssede, and L. Laguerre, "Numerical modeling of guided waves interaction with nonaxisymmetric cracks in elastic cylinders", International Journal of Solids and Structures 48, 764-774 (2011).

${ }^{31}$ G. R. Liu, K. Y. Lam, and Y. Ohyoshi, "A technique for analyzing elastodynamic responses of anisotropic laminated plates to line loads", Composites Part B 28, 667-677 (1997).

32 J. D. Achenbach, Reciprocity in elastodynamics (Cambridge University Press, Cambridge, UK) (2003), 255 p.

33 T. Vogt, M. Lowe, and P. Cawley, "The scattering of guided waves in partly embedded cylindrical structures", Journal of the Acoustical Society of America 113, 12581272 (2003).

34 J. J. Ditri, "Utilization of guided waves for the characterization of circumferential cracks in hollow cylinders", Journal of the Acoustical Society of America 96, 3769-3775 (1994).

35 W. Seemann, A. Ekhlakov, E. Glushkov, N. Glushkova, and O. Kvasha, "The modeling of piezoelectrically excited waves in beams and layered substructures", Journal of Sound and Vibration 301, 1007-1022 (2007).

36 B. Pavlakovic and M. Lowe, "Disperse user's manual", Version 2.0.11, p. 131 (2001).

37 F. Simonetti, "Sound propagation in lossless waveguides coated with attenuative materials", Ph.D. thesis, Imperial College of Science, Technology and Medecine, University of London (2003).

38 D. Clorennec, C. Prada, and D. Royer, "Laser impulse generation and interferometer detection of zero group velocity Lamb mode resonance", Applied Physics Letters 89, 024101 (2006)

39 M. F. Werby and H. Uberall, "The analysis and interpretation of some special properties of higher order symmetric Lamb waves: The case for plates", Journal of the Acoustical Society of America 111, 2686-2691 (2002).

40 A. L. Shuvalov and O. Poncelet, "On the backward Lamb waves near thickness resonances in anisotropic plates", International Journal of Solids and Structures 45, 3430-3448 (2008).

41 A. Gibson and J. S. Popovics, "Lamb wave basis for impact-echo method analysis", Journal of Engineering Mechanics 131, 438-443 (2005).

42 C. Prada, D. Clorennec, and D. Royer, "Local vibration of an elastic plate and zero-group velocity Lamb modes",
Journal of the Acoustical Society of America 124, 203-212 (2008).

43 M. Castaings and M. Lowe, "Finite element model for waves guided along solid systems of arbitrary section coupled to infinite solid media", Journal of the Acoustical Society of America 123, 696-708 (2008).

${ }^{44}$ F. Treyssede, K. L. Nguyen, A. S. Bonnet-BenDhia, and C. Hazard, "On the use of a safe-pml technique for modeling two-dimensional open elastic waveguides", in Proc. Acoustics 2012, 23-27 april, Nantes (France), 667-672 (2012). 